\title{
Nouvelle hausse des cas de mauvais traitements d'enfants
}

\section{Markus Wopmann}

Dr méd., Responsable du Groupe de travail pour la protection de l'enfant, médecin-chef clinique pour enfants et adolescents hôpital cantonal, Baden, membre FMH

En 2016, pour la $8^{\mathrm{e}}$ année consécutive, le nombre d'enfants pris en charge dans les cliniques pédiatriques suisses, en ambulatoire ou stationnaire, suite à de mau-

\section{En 2016, les cliniques pédiatriques ont enregistré deux enfants décédés des suites d'une maltraitance physique.}

vais traitements avérés ou suspectés, a été enregistré. Cette année ce sont les données de 21 des 25 cliniques pédiatriques de Suisse qui ont pu être enregistrées, ce qui représente un feed-back de $84 \%$ des cliniques. Encore une fois, toutes les grandes et moyennes cliniques pédiatriques ont transmis leurs cas, si bien que le nombre de cas que nous avons répertorié représente une très grande partie du nombre de mauvais traitement que les cliniques pédiatriques suisses ont pris en charge durant l'année écoulée.

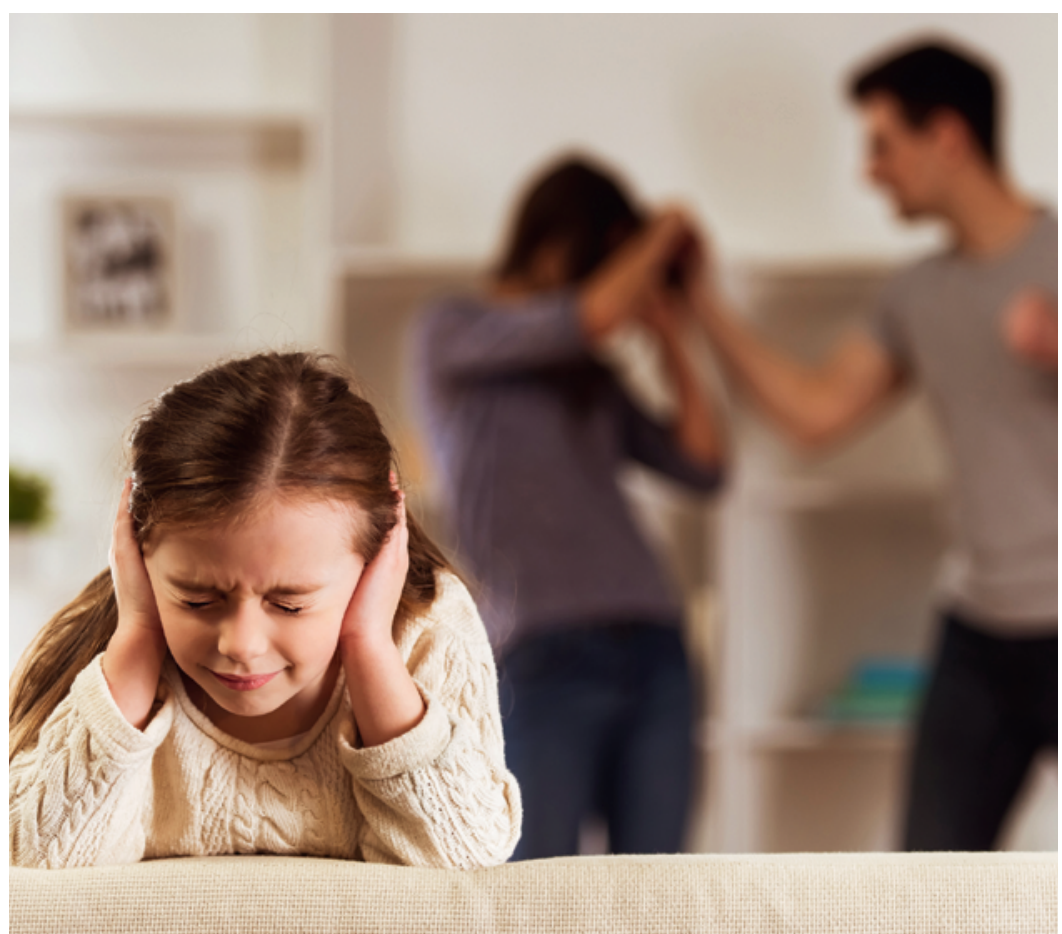

Toujours plus d'enfants perçoivent la violence (souvent répétée) entre les parents avec lesquels ils vivent.

\section{Résultats}

1575 cas au total ont été signalés par les 21 cliniques. Alors que plusieurs cliniques ont recensé moins de cas que l'année précédente et quelques cliniques un nombre pratiquement équivalent, il est apparu dans les cliniques universitaires de Lausanne et de Genève une augmentation massive de cas (>200 cas de plus !). Cette augmentation est à attribuer en première ligne au fait que nouvellement ces cliniques ont également été averties lors d'interventions policières pour des cas de violences domestiques dans des foyers dans lesquels vivaient des enfants. Dans le cadre de cette action, l'état psychologique des enfants a été évalué, qu'ils soient directement ou indirectement touché par la violence domestique. La répartition dans les différents sousgroupes donne les chiffres suivants :

\begin{tabular}{lrr}
\hline Maltraitance physique & 367 & $(23,3 \%)$ \\
\hline Négligence & 319 & $(20,3 \%)$ \\
\hline Maltraitance psychique & 581 & $(36,9 \%)$ \\
\hline Abus sexuel & 306 & $(19,4 \%)$ \\
\hline Syndrome de Münchhausen par procuration & 2 & $(0,1 \%)$
\end{tabular}

Un bon tiers de ces cas concerne des enfants pour qui une maltraitance psychique a été diagnostiquée. Cela s'explique par le fait que toujours plus d'enfants perçoivent la violence (souvent répétée) entre les parents avec lesquels ils vivent, ce qui affecte psychologiquement très durement. Avec $44 \%$ de garçons et $56 \%$ de filles, la répartition selon le genre est pratiquement égale, comme l'an passé. Une maltraitance sur 6 concerne un enfant qui a moins d'une année, près de la moitié des enfants maltraités ont moins de 6 ans.

\begin{tabular}{lll}
\hline $\begin{array}{l}\text { Répartition des sous-groupes } \\
\text { selon le sexe }\end{array}$ & Garçons & Filles \\
\hline Maltraitance physique & $53,4 \%$ & $46,6 \%$ \\
\hline Négligence & $49,5 \%$ & $50,5 \%$ \\
\hline Maltraitance psychique & $47,5 \%$ & $52,5 \%$ \\
\hline Abus sexuel & $19,9 \%$ & $80,1 \%$ \\
\hline
\end{tabular}

Il y a pratiquement un équilibre de genre dans toutes les formes de maltraitance excepté en ce qui concerne 
les abus sexuels pour lesquels les filles sont 4 fois plus touchées que les garçons.

\begin{tabular}{lll}
\hline Certitude du diagnostic & & \\
\hline Sûr & 959 & $(60,9 \%)$ \\
\hline Probable & 242 & $(15,4 \%)$ \\
\hline Pas clair & 273 & $(17,3 \%)$ \\
\hline Aucune indication & 101 & $(6,4 \%)$ \\
\hline
\end{tabular}

Etonnamment le diagnostic de maltraitance psychique a été classifié dans $84,2 \%$ comme sûr, dans les cas de maltraitance physique et de négligence cette certitude n'est donnée que dans seulement la moitié des cas. Les

\section{Une maltraitance sur 6 concerne un enfant qui a moins d'une année, près de la moitié des enfants maltraités ont moins de 6 ans.}

abus sexuels n'ont été jugé comme certains que dans $37,6 \%$ des cas, ce qui reflète également la difficulté de ces diagnostiques.

\begin{tabular}{lrc}
\hline Auteur: relation avec I'enfant & & \\
\hline Famille & 1272 & $(80,8 \%)$ \\
\hline Connu de l'enfant & 186 & $(11,8 \%)$ \\
\hline Extra-familial & 37 & $(2,3 \%)$ \\
\hline Inconnu & 80 & $(5,1 \%)$ \\
\hline
\end{tabular}

Plus de 95\% des cas de maltraitance psychique ou de négligence ont lieu dans le cadre familial, les maltraitances physiques dans $76 \%$ des cas, les abus sexuels dans $39,2 \%$. Près de $20 \%$ des violences sexuelles ont été perpétrées par des auteurs extrafamiliaux ou inconnus.

\begin{tabular}{lll}
\hline Auteur: sexe & & \\
\hline Masculin & 717 & $(45,5 \%)$ \\
\hline Féminin & 384 & $(24,4 \%)$ \\
\hline $\begin{array}{l}\text { Masculin et féminin (en général les deux } \\
\text { parents) }\end{array}$ & 351 & $(22,3 \%)$ \\
\hline Inconnu & 123 & $(7,8 \%)$ \\
\hline
\end{tabular}

Nous retrouvons pratiquement la même répartition que l'an passé. Comme on peut s'y attendre, dans le domaine des abus sexuels, la proportion des auteurs masculins est la plus haute avec $87,3 \%$. Les hommes représentent également la plus grande part des auteurs en ce qui concerne les maltraitances physiques et les maltraitances psychologiques avec respectivement $41,4 \%$ et $44,8 \%$. C'est seulement pour les cas de négli- gence que la proportion des auteurs femmes est sensiblement plus élevée que celle des auteurs hommes.

\begin{tabular}{lrl}
\hline Auteur: âge & & \\
\hline Plus de 18 ans & 1343 & $(85,3 \%)$ \\
\hline Moins de 18 ans & 147 & $(9,3 \%)$ \\
\hline Moins et plus de 18 ans (plusieurs auteurs) & 9 & $(0,6 \%)$ \\
\hline Âge inconnu / pas d'indication & 76 & $(4,8 \%)$ \\
\hline
\end{tabular}

Les jeunes auteurs sont responsables des abus sexuels dans $27,5 \%$ du nombre total de cas et des maltraitances physique dans 10\% des cas. Dans les autres formes de maltraitance leur proportion est insignifiante.

\begin{tabular}{|c|c|c|}
\hline \multicolumn{3}{|l|}{$\begin{array}{l}\text { Annonce à l'autorité de protections de l'enfant } \\
\text { et de l'adulte }\end{array}$} \\
\hline Déjà faite par une autre instance & 268 & $(17,0 \%)$ \\
\hline Par le groupe de protection de l'enfant & 419 & $(26,6 \%)$ \\
\hline $\begin{array}{l}\text { Recommandée par le groupe de protection } \\
\text { de l'enfant }\end{array}$ & 119 & $(7,6 \%)$ \\
\hline \multicolumn{3}{|l|}{ Annonce à l'autorité judiciaire } \\
\hline Déjà faite par une autre instance & 214 & $(13,6 \%)$ \\
\hline Par le groupe de protection de l'enfant & 79 & $(5,0 \%)$ \\
\hline $\begin{array}{l}\text { Recommandée par le groupe de protection } \\
\text { de l'enfant }\end{array}$ & 72 & $(4,6 \%)$ \\
\hline
\end{tabular}

\section{Conclusion}

- La croissance continue des cas de mauvais traitements d'enfants enregistrée par les cliniques pédiatriques suisses atteste de la triste importance de ces diagnostiques.

- La maltraitance psychologique, avant tout due à la violence entre les parents dans le lieu de vie, est un phénomène qui est de plus en plus observé. Malheureusement dans beaucoup de familles deux interventions policières ou plus sont enregistrées dans une même année, ce qui souligne encore plus l'importance de cette thématique.

- En 2016, les cliniques pédiatriques ont enregistré deux enfants décédés des suites d'une maltraitance physique. Un de ces enfants était âgé de moins d'un an, le deuxième avait entre un et deux ans. Cela correspond aux expériences internationales qui montrent que le risque de maltraitance sévère ou même mortelle sur les très jeunes enfants est au plus haut.

Crédit illustration

(c) Vadimgozhda | Dreamstime.com 